http://dx.doi.org/10.18778/2196-8403.2007.17

\title{
THEMATISCHER SCHWERPUNKT 2009: Dialog der Germanisten in Zeiten der Teilung
}

Mit dieser Thematik wird z.T. an das Schwerpunktthema von 2005 „Fachgeschichte problemorientiert" angeknüpft, wobei nun die Fachgeschichte in einer breiteren internationalen Perspektive gesehen werden soll. Trotz der durch den Eisernen Vorhang bedingten politischen und wirtschaftlichen Teilung Europas (und speziell der durch Mauer und Stacheldraht besonders rigorosen Teilung der beiden deutschen Staaten) hat es vor 1989 nicht an zuweilen erfolgreichen Versuchen gefehlt, im Bereich der Kultur, also der Kunst und Wissenschaft, Dialoge über die starren Grenzen hinweg zu führen, um Ideen, Methoden und Erfahrungen auszutauschen. Das geschah auch in der Germanistik, in der Sprachwissenschaft nicht weniger als in der Literaturwissenschaft, und zwar ebenso auf großen Tagungen wie denen der Internationalen Germanisten-Vereinigung wie auf kleineren Konferenzen, bei mehr oder weniger zufälligen Begegnungen in neutralen Ländern und durch persönliche Kontakte, die freilich nicht selten von staatlicher Seite behindert wurden. Dass Germanisten auch mal die Fronten wechselten (fast immer von Ost nach West), konnte den Dialog sowohl fördern als auch stören.

Das Loch in der Mauer. Der innerdeutsche Literaturaustausch heißt ein 1997 erschienenes, von Mark Lehmstedt und Siegfried Lokatis herausgegebenes Buch, in dem es hauptsächlich um Institutionen, um Verlags- und AutorenBeziehungen, punktuell aber auch um die Germanistik geht. Die dort angedeutete Perspektive einer Fachgeschichte ist zu erweitern: Wie funktionierten die innerdeutschen Dialoge der Germanisten im Einzelnen? Wie groß war das Loch, und wer nutzte es wie und warum? Vor allem aber soll die räumliche Beschränkung aufgehoben und gefragt werden: Wie groß war der Riss im Eisernen Vorhang, der es auch Germanisten diesseits und jenseits der monströsen Grenzen erlaubte, die Teilung (etwa die zwischen Polen und der Bundesrepublik Deutschland) gelegentlich im Dialog zu überwinden? Welche Ergebnisse zeitigten Austausch und Zusammenarbeit hier und da, in früheren und späteren Jahren?

Die Convivium-Redaktion lädt zu einer kritischen Bestandsaufnahme einer wichtigen Epoche der germanistischen Fachgeschichte ein. Auch die Mitteilung persönlicher Erfahrungen, die nicht vorrangig einen wissenschaftlichen Bezug haben, sowie Interviews mit Zeitzeugen sind sehr willkommen. Wer immer einen Beitrag zu diesem thematischen Schwerpunkt verfassen möchte, wird gebeten, der Redaktion den vorläufigen Titel bis zum 30. Juni 2008 mit- 
zuteilen und den Beitrag auf Diskette (mit zwei Ausdrucken) bis zum 30. November 2008 an die im Impressum genannte Redaktionsanschrift zu senden. Für die formale Gestaltung der Artikel sind die „Hinweise zur Einrichtung des druckfertigen Manuskripts" verbindlich, über die die Convivium-Website convivium.pl informiert. 